\title{
Ghost free mimetic massive gravity
}

\author{
Ali H. Chamseddine ${ }^{a, b}$ and Viatcheslav Mukhanov ${ }^{c, d, e}$ \\ ${ }^{a}$ Physics Department, American University of Beirut, \\ Beirut, Lebanon \\ ${ }^{b}$ I.H.E.S., \\ F-91440 Bures-sur-Yvette, France \\ ${ }^{c}$ Theoretical Physics, Ludwig Maxmillians University, \\ Theresienstr. 37, 80333 Munich, Germany \\ ${ }^{d} M P I$ for Physics, \\ Foehringer Ring, 6, 80850, Munich, Germany \\ e LPT de l'Ecole Normale Superieure, \\ 24 rue Lhomond, 75231 Paris cedex, France \\ E-mail: chams@aub.edu.lb, vilatcheslav.mukhanov@lmu.de
}

ABSTRACT: The mass of the graviton can be generated using a Brout-Englert-Higgs mechanism with four scalar fields. We show that when one of these fields is costrained as in mimetic gravity, the massive gravity obtained is ghost free and consistent. The mass term is not of the Fierz-Pauli type. There are only five degrees of freedom and the sixth degree of freedom associated with the Boulware-Deser ghost is constrained and replaced by mimetic matter to all orders. The van Dam-Veltman-Zakharov discontinuity is also absent.

Keywords: Classical Theories of Gravity, Effective Field Theories

ARXIV EPRINT: 1805.06283 
In [1] we have proposed an alternative formulation of gravity explicitly isolating the scale factor in the physical metric $g_{\mu \nu}$ by rewriting it as

$$
g_{\mu \nu}=\tilde{g}_{\mu \nu}\left(\tilde{g}^{\kappa \lambda} \partial_{\kappa} \phi \partial_{\lambda} \phi\right),
$$

where $\tilde{g}_{\mu \nu}$ is some auxiliary metric and $\phi$ is a scalar field. It follows from (1) that $\phi$ must obey the constraint

$$
g^{\mu \nu} \partial_{\mu} \phi \partial_{\nu} \phi=1
$$

Considering $\tilde{g}_{\mu \nu}$ as a fundamental variable in the Einstein-Hilbert action we find that Einstein equations are modified to

$$
G_{\mu \nu}-T_{\mu \nu}-(G-T) \partial_{\mu} \phi \partial_{\nu} \phi=0
$$

where $G_{\mu \nu}$ and $T_{\mu \nu}$ are, respectively, the Einstein tensor and the energy-momentum tensor for matter. We use the units in which $8 \pi G=1$. The trace part of equation (3) vanishes identically as a consequence of scale invariance $\tilde{g}_{\mu \nu} \rightarrow \Omega_{\mu \nu}^{2} \tilde{g}$ for the auxiliary metric $\tilde{g}_{\mu \nu}$ in equation (2). As a result the modified Einstein equations (2) and (3) have an extra solution even in the absence of matter which could mimic cold dark matter in our Universe. As it was noticed in [2] this mimetic gravity is fully equivalent to Einstein gravity with the extra constraint (2) implemented in the action using a Lagrange multiplier. The field $\phi$ can be taken in the synchronous coordinate system, solving constraint (2), as time coordinate. It becomes dynamical only when combined with the longitudinal mode of gravity. The proposed model does not only give a plausible explanation for the origin of dark matter but also provides a new approach to resolve singularities in General Relativity [3, 4]. Moreover, the appearance of the constrained field $\phi$ has found a justification in the noncommutative approach to the quanta of geometry $[5,6]$.

In this letter we will consider the theory of mimetic massive gravity and show that this theory is ghost free to all orders and describes the massive graviton with five degrees of freedom which are completely decoupled from mimetic matter in the linear approximation.

The simplest way of giving mass to the graviton without explicitly spoiling diffeomorphism invariance, reflecting the freedom in the choice of coordinate system, is by employing the Brout-Englert-Higgs mechanism with four scalar fields $\phi^{A}, A=0,1,2,3$ [7-9]. In Minkowski space-time the broken symmetry phase,

$$
\left\langle\phi^{A}\right\rangle=\delta_{\mu}^{A} x^{\mu}=x^{A}
$$

is degenerate with respect to a vacuum choice up to Poincare transformations. For small perturbations of the fields

$$
\phi^{A}=x^{A}+\chi^{A},
$$

the three scalars $\chi^{i}$ are absorbed to give mass to the graviton, while the fourth field $\chi^{0}$ leads to a ghost, unless the mass term in the Lagrangian is taken to be of the FierzPauli form [10] where this ghost is not excited at the linear level. However, generically, it reappears as a nonlinear Boulware-Deser ghost on non-trivial backgrounds [11]. The idea we put forward in this letter, is to use as one of the four fields, needed for providing mass 
to the graviton, the mimetic field $\phi \equiv \phi^{0}$. Because this field is constrained to be always in the broken symmetry phase the dangerous degree of freedom is thus replaced by dark matter and the ghost is avoided to any order in perturbation theory. As we show below the mass term in this case must be necessarily taken to be different from Fierz-Pauli type.

In massive gravity a central role is played by the induced metric perturbations

$$
\bar{h}^{A B}=g^{\mu \nu} \partial_{\mu} \phi^{A} \partial_{\nu} \phi^{B}-\eta^{A B}
$$

the components of which are scalars under diffeomorphism transformations. We will raise and lower capital indices with the help of the auxiliary Minkowski metric $\eta^{A B}=$ $(1,-1,-1,-1)$, so that, for example, $\bar{h} \equiv \bar{h}_{A}^{A}=\eta_{A B} \bar{h}^{A B}$.

We consider the theory with action

$$
I=\int d^{4} x \sqrt{g}\left(-\frac{1}{2} R+\frac{m^{2}}{8}\left(\frac{1}{2} \bar{h}^{2}-\bar{h}^{A B} \bar{h}_{A B}\right)+\lambda\left(g^{\mu \nu} \partial_{\mu} \phi^{0} \partial_{\nu} \phi^{0}-1\right)\right),
$$

where the last term accounts for the mimetic origin of $\phi^{0}$ and the mass term has relative coefficient $\frac{1}{2}$ between $\bar{h}^{2}$ and $\bar{h}^{A B} \bar{h}_{A B}$ in distinction from Fierz-Pauli term where this coefficient is 1 . The reason for this choice will become clear later. The equations of motion are obtained, first by varying with respect to $\delta g^{\mu \nu}$ :

$$
\begin{aligned}
G_{\mu \nu}= & -\frac{m^{2}}{8}\left(\frac{1}{2} \bar{h}^{2}-\bar{h}^{A B} \bar{h}_{A B}\right) g_{\mu \nu}+\lambda\left(2 \partial_{\mu} \phi^{0} \partial_{\nu} \phi^{0}\right) \\
& +\frac{m^{2}}{2}\left(\frac{1}{2} \bar{h} \partial_{\mu} \phi_{A} \partial_{\nu} \phi^{A}-\bar{h}_{A B} \partial_{\mu} \phi^{A} \partial_{\nu} \phi^{B}\right),
\end{aligned}
$$

and next with respect to $\delta \phi^{A}$ :

$$
\nabla^{\mu}\left(m^{2}\left(\frac{1}{2} \bar{h} \partial_{\mu} \phi_{A}-\bar{h}_{A B} \partial_{\mu} \phi^{B}\right)+4 \lambda \delta_{0 A} \partial_{\mu} \phi^{0}\right)=0 .
$$

Varying with respect to $\delta \lambda$ gives:

$$
\bar{h}^{00}=0 \text {. }
$$

Let us consider small perturbations around Minkowski background, $g_{\mu \nu}=\eta_{\mu \nu}+h_{\mu \nu}$ and $\phi^{A}=x^{A}+\chi^{A}$, and linearize the above equations in $h_{\mu \nu}$ and $\chi^{A}$ keeping in mind that $\lambda$ is of first order in perturbations. In equation (10) we first set $A=0$ to get

$$
\partial_{0} \lambda-\frac{m^{2}}{4}\left(\partial^{\rho} \bar{h}_{\rho 0}-\frac{1}{2} \partial_{0} \bar{h}\right)=0,
$$

and then $A=k$ to obtain

$$
m^{2}\left(\partial^{\rho} \bar{h}_{\rho k}-\frac{1}{2} \partial_{k} \bar{h}\right)=0 .
$$

The linearized Einstein tensor in $h_{\mu \nu}$ is equal to

$$
\begin{aligned}
G_{\mu \nu}\left(h_{\rho \sigma}\right)= & -\frac{1}{2}\left(\partial^{2} h_{\mu \nu}-\partial_{\mu} \partial^{\rho} h_{\rho \nu}-\partial_{\nu} \partial^{\rho} h_{\rho \mu}+\partial_{\mu} \partial_{\nu} h\right) \\
& +\frac{1}{2} \eta_{\mu \nu}\left(\partial^{2} h-\partial^{\sigma} \partial^{\rho} h_{\rho \sigma}\right),
\end{aligned}
$$


where $\partial^{2} \equiv \partial^{\mu} \partial_{\mu} \equiv \square$ and $h \equiv \eta^{\mu \nu} h_{\mu \nu}$. To first order in perturbations

$$
\bar{h}^{A B}=\delta_{\mu}^{A} \delta_{\nu}^{B} h^{\mu \nu}+\partial^{A} \chi^{B}+\partial^{B} \chi^{A},
$$

where $h^{\mu \nu}=g^{\mu \nu}-\eta^{\mu \nu}$. Because $g^{\mu \sigma} g_{\sigma \nu}=\delta_{\nu}^{\mu}$ it follows that in linear order $h^{\mu \nu}=$ $-\eta^{\mu \sigma} \eta^{\nu \rho} h_{\sigma \rho}$. Now keeping in mind that capital indices are moved with Minkowski metric $\eta_{A B}$ and replacing them by Greek indices from (14) we find that

$$
h_{\mu \nu}=-\bar{h}_{\mu \nu}+\partial_{\mu} \chi_{\nu}+\partial_{\nu} \chi_{\mu} .
$$

Substituting (15) in (13) we find that all $\chi$ terms cancel and hence $G_{\mu \nu}\left(h_{\rho \sigma}\right)=-G_{\mu \nu}\left(\bar{h}_{\rho \sigma}\right)$, that is the linearized Einstein tensor can be expressed entirely in terms of gauge invariant variables $\bar{h}_{\rho \sigma}$ as it must be. Then taking into account that $\bar{h}_{00}=0$ due to constraint (10) the linearized Einstein equations take the form

$$
\begin{aligned}
G_{00}\left(-\bar{h}_{\rho \sigma}\right) & =2 \lambda+\frac{m^{2}}{4} \bar{h}, \\
G_{0 i}\left(-\bar{h}_{\rho \sigma}\right) & =-\frac{m^{2}}{2} \bar{h}_{0 i}, \\
G_{i j}\left(-\bar{h}_{\rho \sigma}\right) & =-\frac{m^{2}}{2}\left(\bar{h}_{i j}-\frac{1}{2} \eta_{i j} \bar{h}\right),
\end{aligned}
$$

where $G_{\mu \nu}\left(-\bar{h}_{\rho \sigma}\right)$ are given in (13) with $h_{\rho \sigma}$ replaced by $-\bar{h}_{\rho \sigma}$. It is easy to see that equations (11) and (12) follow from the ten equations (16), (17), (18) as a consequence of Bianchi identities $\partial^{\mu} G_{\mu \nu}=0$. Ten equations are enough to determine all ten unknown variables $\lambda, \bar{h}_{0 i}$ and $\bar{h}_{i j}$ (recall that $\bar{h}_{00}=0$ due to mimetic constraint). Let us start with $i-j$ equations and first simplify $G_{i j}\left(-\bar{h}_{\mu \nu}\right)$. Using equation (12) we find that

$$
\partial_{i} \partial^{\rho} \bar{h}_{\rho k}+\partial_{k} \partial^{\rho} h_{\rho i}=\partial_{i} \partial_{k} \bar{h},
$$

and

$$
\partial^{\sigma} \partial^{\rho} \bar{h}_{\rho \sigma}=\partial^{0}\left(\partial^{\rho} \bar{h}_{\rho 0}\right)+\partial^{k}\left(\partial^{\rho} \bar{h}_{\rho k}\right)=\frac{1}{2} \partial^{2} \bar{h}+\frac{4 \ddot{\lambda}}{m^{2}},
$$

where we have used (11) to express $\partial^{\rho} \bar{h}_{\rho 0}$ in terms of $\bar{h}$ and $\lambda$ and dot denotes time derivative. Taking this into account the $i-j$ equations (18) become

$$
\partial^{2} \bar{h}_{i j}-\eta_{i j}\left(\frac{1}{2} \partial^{2} \bar{h}-\frac{4 \ddot{\lambda}}{m^{2}}\right)=-m^{2}\left(\bar{h}_{i j}-\frac{1}{2} \eta_{i j} \bar{h}\right) .
$$

It immediately follows from (21) that the traceless part of spatial metric components

$$
\bar{h}_{i j}^{T} \equiv \bar{h}_{i j}-\frac{1}{3} \eta_{i j} \bar{h}
$$

satisfy the wave equation

$$
\left(\square+m^{2}\right) \bar{h}_{i j}^{T}=0,
$$

which describes the massive graviton with five degrees of freedom. The $0-0$ equation (16) gives

$$
\Delta \bar{h}+\partial^{i} \partial^{j} \bar{h}_{i j}=4 \lambda+\frac{m^{2}}{2} \bar{h},
$$


where $\triangle=-\partial^{i} \partial_{i}$, when combined with (22) allows us to express $\bar{h}$ just in terms of $\lambda$ and $\bar{h}_{i j}^{T}$

$$
\bar{h}=6\left(\frac{\partial^{i} \partial^{j} \bar{h}_{i j}^{T}-4 \lambda}{3 m^{2}-4 \triangle}\right) .
$$

Substituting this expression into the trace of equation (21),

$$
\left(\square+m^{2}\right) \bar{h}-\frac{24}{m^{2}} \ddot{\lambda}=0,
$$

and taking into account that $\bar{h}_{i j}^{T}$ satisfy (23) we obtain the equation which describes mimetic matter

$$
\ddot{\lambda}+\frac{m^{2}}{4} \lambda=0 .
$$

Finally to determine $\bar{h}_{0 i}$ we need $0-i$ Einstein equations. To simplify them we use equation (11) to express $\partial_{i} \partial^{\rho} \bar{h}_{\rho 0}$ in (13) in terms of $\bar{h}$ and $\lambda$. As a result equation (17) takes the form

$$
\Delta \bar{h}_{0 i}+\partial_{0} \partial^{k} \bar{h}_{k i}+\partial_{0} \partial_{i}\left(\frac{4}{m^{2}} \lambda-\frac{1}{2} \bar{h}\right)=m^{2} \bar{h}_{0 i},
$$

from which, using (25) and (22), one gets

$$
\bar{h}_{0 i}=\frac{\partial_{0} \partial^{k} \bar{h}_{k i}^{T}}{m^{2}-\Delta}-\frac{\partial_{0} \partial_{i}}{m^{2}-\Delta}\left(\frac{\partial^{l} \partial^{m} \bar{h}_{l m}^{T}}{3 m^{2}-4 \triangle}\right)+\frac{16 \partial_{0} \partial_{i} \lambda}{m^{2}\left(3 m^{2}-4 \triangle\right)} .
$$

Thus we have found that massive mimetic gravity describes a massive graviton characterized by the traceless part of $\bar{h}_{k i}^{T}$ obeying equation (23) and mimetic matter described by $\lambda$, which satisfies (27). The remaining variables $\bar{h}$ and $\bar{h}_{0 i}$ are entirely expressed in terms of $\bar{h}_{k i}^{T}$ and $\lambda$ (see (25) and (29)). Mimetic matter is modified and instead of being imitating dust behaves like particles at rest (with zero momentum) of mass equal to half of the graviton mass. In the models of massive gravity usually considered with Fierz-Pauli mass term, corresponding to a combination of $\bar{h}^{2}-\bar{h}^{A B} \bar{h}_{A B}$, Bianchi identities enforce the vanishing of the perturbations of the scalar curvature $\delta R=0$. In turn this leads to vDVZ discontinuity $[12,13]$, which is resolved only after taking into account the nonlinear corrections [14]. In our case the Bianchi identities impose conditions (11) and (12) which are similar to the harmonic gauge choice, although here these equations are gauge invariant and do not depend on a coordinate system. Therefore, vDVZ discontinuity is already absent at the linear level. In other models considered before, the mass term we used leads to a ghost mode, which is manifested by the dynamics of the $h_{00}$ metric component in the linear theory. In our theory this mode is constrained and replaced by mimetic matter, which can also explain the observed dark matter in the universe. This guarantees that mimetic massive gravity is fully ghost free to all orders [15]. Moreover, in linear order the mimetic matter is completely decoupled from the graviton.

To consider the massless limit it is convenient to decompose $\bar{h}_{k i}^{T}$ in irreducible pieces with respect to the rotation group $S O(3)$

$$
\bar{h}_{k i}^{T}=\left(\partial_{k} \partial_{i}+\frac{1}{3} \eta_{k i} \Delta\right) S+\partial_{k} V_{i}+\partial_{i} V_{k}+\bar{h}_{k i}^{T T},
$$


where $V_{i}$ is transverse $\partial^{i} V_{i}=0$ and $\bar{h}_{k i}^{T T}$ is not only traceless but also transverse $\partial^{i} \bar{h}_{k i}^{T T}=0$. Thus, the five degrees of freedom of massive graviton are represented by one scalar mode $S$, two vector modes $V_{i}$ and two tensor modes $\bar{h}_{k i}^{T T}$. In [15] we study the dynamics of these modes separately and by considering quantum fluctuations show that nonlinear corrections for the scalar and vector modes become important at the energy scale of order $m^{1 / 2}$. At this scale, they get in strongly coupled regime and decouple from two transverse degrees of freedom of the graviton which become strongly coupled only at the Planck scale. Thus, at energies above $m^{1 / 2}$ the graviton has only two propagating degrees of freedom.

\section{Acknowledgments}

The work of A. H. C is supported in part by the National Science Foundation Grant No. Phys-1518371. The work of V.M. is supported in part by The Dark Universe and the Cluster of Excellence EXC 153 Origin and Structure of the Universe. V.M. thanks ENS, where a part of this work was completed, for hospitality.

Open Access. This article is distributed under the terms of the Creative Commons Attribution License (CC-BY 4.0), which permits any use, distribution and reproduction in any medium, provided the original author(s) and source are credited.

\section{References}

[1] A.H. Chamseddine and V. Mukhanov, Mimetic dark matter, JHEP 11 (2013) 135 [arXiv: 1308.5410] [INSPIRE].

[2] A. Golovnev, On the recently proposed mimetic dark matter, Phys. Lett. B 728 (2014) 39 [arXiv:1310.2790] [INSPIRE].

[3] A.H. Chamseddine and V. Mukhanov, Resolving cosmological singularities, JCAP 03 (2017) 009 [arXiv: 1612.05860] [INSPIRE].

[4] A.H. Chamseddine and V. Mukhanov, Nonsingular black hole, Eur. Phys. J. C 77 (2017) 183 [arXiv: 1612.05861] [INSPIRE].

[5] A.H. Chamseddine, A. Connes and V. Mukhanov, Quanta of geometry: noncommutative aspects, Phys. Rev. Lett. 114 (2015) 091302.

[6] A.H. Chamseddine, A. Connes and V. Mukhanov, Geometry and the quantum: basics, JHEP 12 (2014) 098 [arXiv: 1411.0977] [INSPIRE].

[7] W. Siegel, Hidden gravity in open string field theory, Phys. Rev. D 49 (1994) 4144 [hep-th/9312117] [INSPIRE].

[8] G. 't Hooft, Unitarity in the Brout-Englert-Higgs Mechanism for Gravity, arXiv:0708.3184 [INSPIRE].

[9] A.H. Chamseddine and V. Mukhanov, Higgs for graviton: simple and elegant solution, JHEP 08 (2010) 011 [arXiv: 1002.3877] [INSPIRE].

[10] M. Fierz and W. Pauli, On relativistic wave equations for particles of arbitrary spin in an electromagnetic field, Proc. Roy. Soc. Lond. A 173 (1939) 211 [InSPIRE]. 
[11] D.G. Boulware and S. Deser, Can gravitation have a finite range?, Phys. Rev. D 6 (1972) 3368 [INSPIRE].

[12] H. van Dam and M.J.G. Veltman, Massive and massless Yang-Mills and gravitational fields, Nucl. Phys. B 22 (1970) 397 [inSPIRE].

[13] V.I. Zakharov, Linearized gravitation theory and the graviton mass, JETP Lett. 12 (1970) 312 [Pisma Zh. Eksp. Teor. Fiz. 12 (1970) 447] [InSPIRE].

[14] A.I. Vainshtein, To the problem of nonvanishing gravitation mass, Phys. Lett. 39B (1972) 393 [INSPIRE].

[15] A.H. Chamseddine and V. Mukhanov, Mimetic massive gravity: beyond linear approximation, arXiv:1805.06598 [INSPIRE]. 